\title{
Grats

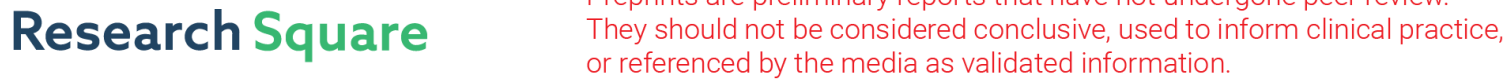 \\ Expression of Five Target Proteins Related to Human Cytomegalovirus Infection in Brain Metastases of Lung Cancer
}

\section{Jie Wu}

The Affiliated Suzhou Science and Technology Town Hospital of Nanjing Medical University

\section{Haifeng Zhao}

The Affiliated Suzhou Science and Technology Town Hospital of Nanjing Medical University

\section{Ke Yan}

The Affiliated Suzhou Science and Technology Town Hospital of Nanjing Medical University

\section{Xiangtong Xie}

Suzhou Kowloon Hospital Shanghai Jiao Tong University School of Medicine

\section{Donghua Gu}

The Affiliated Suzhou Science and Technology Town Hospital of Nanjing Medical University

\section{Weihua Wang}

The Affiliated Suzhou Science and Technology Town Hospital of Nanjing Medical University

\section{Xifeng Fei}

Suzhou Kowloon Hospital Shanghai Jiao Tong University School of Medicine

\section{Wenyu Zhu ( $\nabla$ wenyuzhu0702@hotmail.com )}

The Affiliated Suzhou Science \& Technology Town Hospital Town Hospital of Nanjing Medical University

\section{Zhimin Wang}

Suzhou Kowloon Hospital Shanghai Jiao Tong University School of Medicine

\section{Qiang Huang}

Second Affiliated Hospital of Soochow University

\section{Research}

Keywords: brain metastases of lung cancer, GBP4, CXCR4, EGFR, TTF1, IE, human cytomegalovirus infection.

Posted Date: July 19th, 2021

DOI: https://doi.org/10.21203/rs.3.rs-707732/v1 
License: (c) (i) This work is licensed under a Creative Commons Attribution 4.0 International License. Read Full License 


\section{Abstract}

Background: The links between brain metastases of lung cancer and human cytomegalovirus (HCMV) infection have been controversial for a long time. This study aims to explore the links between brain metastases of lung cancer and HCMV infection from the perspective of expression and detection of HCMV immediate early gene (IE), guanine nucleotide-binding protein 4 (GBP4), CXC chemokine receptor 4 (CXCR4), thyroid transcription factor 1 (TTF1) and epidermal growth factor receptor (EGFR) proteins.

Methods: We collected brain metastases specimens and lung primary tumor specimens of a series of patients that have not undergone any treatment. Conventional hematoxylin and eosin staining and immunohistochemical staining of target molecules was performed. We used the ImageJ software to process the average optical density value of immune complexes and GraphPad Prism 8.0.1 to perform image analysis, and the SPSS 22.0 statistics package ( $t$ test) to analyze the expression differences of target molecules.

Results: Based on five cases of brain metastases and two cases of lung primary tumors, a total of seven samples were investigated. Conventional pathology diagnosis reported four cases of brain metastases of lung adenocarcinoma and one case of brain metastases of mixed small cell lung cancer with adenocarcinoma. Among the 19 molecular immunopathological test samples, only GBP4, related to HCMV infection, and TTF1, related to metastases, were highly expressed in all seven samples. A comparison of the AOD values of the primary lung cancer to the AOD values of brain metastases, yielded statistically significant differences as follows: in Case No.1, GBP4 ( $p=0.016)$, EGRF ( $<<0.001)$; in Case No. 2 , IE $(p<0.001), \operatorname{CXCR} 4(p=0.005), \operatorname{EGFR}(p=0.023), \operatorname{TTF1}(p=0.004)$.

Conclusions: Although TTF1 is known to be a kinesin for brain metastases of lung cancer cells and it is associated with poor survival prognosis, the role of GBP4, which is closely related to HCMV infection and a key protein of brain metastases of lung cancer, remains unknown.

The findings provide new knowledge into the role of GBP4 and could provide clues for devising novel strategies for target molecular therapy research in brain metastases of lung cancer in the context of HCMV infection.

\section{Background}

About $10 \%-20 \%$ of cancers metastasize to the brain, with the 2-year and 5-year survival rates after the initial diagnosis of the primary cancer being $8.1 \%$ and $2.4 \%$, respectively [1]. These low survival rates are mainly attributed to the limited effectiveness of conventional surgery, radiotherapy and chemotherapy. More recently, targeted therapy has become a new option to improve the prognosis of patients with brain metastases of lung cancer. Although still preliminary, the optimization of more critical target molecules has become a hot research topic and a promising new therapeutic avenue for these patients. Key molecules expressed in brain metastases of lung cancer include thyroid transcription factor 1 (TTF1) and epidermal growth factor receptor (EGFR) [2]. When infection with human cytomegalovirus and brain 
metastases of lung cancer co-occur, the complexity of the condition increases dramatically. To the best of our knowledge, this is the first study investigating the association between HCMV and brain metastases of lung cancer. Previous research had focused on the study of HCMV in brain metastases of breast cancer and colorectal cancer [3]. Prior studies on HCMV carcinogenesis mainly included the HCMV immediate early gene (IE) [4], CXC chemokine receptor 4 (CXCR4) [5], and the guanine nucleotide-binding protein 4 (GBP4) [6] encoded by antiviral interferon. However, the role of these three genes in brain metastases of lung cancer is poorly understood, and has not been described in the TCGA and GEO cancer databases it would be important to provide references or links for the TCGA and GEO cancer databases.

We have built on previous knowledge of TTF1 and EGFR in brain metastases of lung cancer $[2,7,8]$. We performed extensive literature searches, given preliminarily evidence that GBP4 could be used as a key regulatory protein of HCMV infection in brain metastases of lung cancer. As a control, we have investigated the expression of a total of five target proteins including IE, GBP4 and CXCR4, along with other 14 proteins reported in molecular pathology routines, in five cases of brain metastases of lung cancer and primary tumors using immunohistochemistry techniques. Statistics were performed on immune complex average optical density (AOD) values. The findings reported here could provide new insights for developing new strategies for target molecular therapy research in brain metastases of lung cancer in the context of HCMV infection.

\section{Materials And Methods}

\section{Materials}

Five cases of brain metastases of lung cancer who underwent intracranial tumor resection in our department between Jan, 2018 and Dec, 2019 and whose diagnosis had been confirmed by conventional pathology were selected. We have also included two cases of lung primary tumors, that had been previously embedded in paraffin wax after resection. Immunohistochemical staining using antibodies for IE and CXCR4 (Santa Cruz, USA), for GBP4 (Shanghai Shenggong), for TTF1 (Guangzhou Anbiping) and for EGFR (Guangzhou Anbiping) was performed simultaneously, as described below. A microtome (Leica, Germany) and an optical microscope (Olympus, Japan) were used for tissue sectioning and imaging.

\section{H\&e And Immunohistochemical Staining Of Paraffin Sections}

All specimens were fixed with $3.7 \%$ neutral buffered formaldehyde solution, routinely dehydrated, embedded in paraffin and sectioned at a thickness of $4 \mu \mathrm{m}$. Hematoxylin and eosin (H\&E) and immunohistochemical staining was performed. The antibody concentrations were as follows: CXCR4 (1:200), GBP4 (1:300) and IE (1:200). TTF1 (ready-to-use) and EGFR (ready-to-use) were detected by a fully automatic immunohistochemistry detector (Leica, Germany). The staining protocol followed the 
manufacturer's instructions. A positive control and phosphate buffered saline (PBS) negative control were performed in same tissue section.

Analysis of average optical density (AOD) of Immune complexes

Paraffin sections were stained by immunohistochemistry and directly observed under a light microscope at $400 \mathrm{X}$ magnification. Immune complexes were identified by their dark brown color. Three fields of view were randomly selected from each section. Images were captured by [author please describe how images were taken] and ImageJ software [author: please indicate the version used] was used to calculate the AOD of the dark brown (positive) immune complexes in each image. To analyze differences in the expression of marker molecules we have perform a t-test on the AOD results using the SPSS 22.0 statistical analysis software. We considered a statistically significant difference at a $\mathrm{P}<0.05$ value. We used the GraphPad Prism7 software to draw the AOD columns figures (see Figs. 3 and 5).

\section{Results}

\section{Conventional pathology}

To characterize the histological cancer type, H\&E stained sections were visualized using the light microscope. Out of the five brain metastases cases, four cases were adenocarcinoma (including two cases of poorly differentiated adenocarcinoma) and one case was of small cell lung carcinoma. In two of these cases we were able to trace back the pathology of lung primary tumor (lung adenocarcinoma and mixed cancer). Mixed cancers are mainly characterized by the presence of glandular cells and small round cells, which tend to be distributed in different areas, as shown in Fig. $1 \mathrm{G}$.

\section{Molecular Pathology}

As part of routine molecular diagnosis, the pathology department of our hospital reports protein expressions in specimens of brain metastases of lung cancer to clinicians. These data are listed in Table 1. Additionally, in order to investigate the levels of expression of five target proteins (IE, GBP4, CXCR4, TTF1 and EGFR) by means of intensity, we have not only performed the layered markings in Table 1, but we have also performed imaging, and compared AOD values using (see Figs. 2-5).

As shown in Table 1, Fig. 2 and Fig. 4, out of the five proteins, only GBP4 protein was highly expressed in both primary tumors and metastases. TTF1 was also highly expressed in Case No. 1-4, but no obvious expression was detected in Case No. 5. As shown in Fig. 3, although GBP4 and TTF1 were both highly expressed, there were differences in their levels of expression. These differences may be attributed to the different pathologies of the tumors. For example, Case No.1 is small cell lung cancer, while Case No. 2 is non-small cell lung carcinoma. We have found that CXCR4 is expressed to various degrees in all cases, however, although its expression is noticeably regional. EGFR was only moderately or highly expressed in some brain metastases (Cases No. 1 and 5), but, at least based on the cases we analyzed, its expression 
levels in the primary tumor seem lower. Although the IE protein was expressed in metastases in our samples, its expression was of low intensity. Based on a qualitative evaluation under the microscopy, along with quantitative data based on statistics (by pairwise comparison) performed on AOD values, the expression intensity of the five analyzed target proteins on four samples of lung primary tumors and brain metastases, was a following: in Case No. 1, GBP4 ( $P=0.016)$, EGRF $(P<0.001)$; in Case No. 2, IE ( $P$ $<0.001)$, CXCR4 $(P=0.005)$, EGFR $(P=0.023)$, TTF1 $(P=0.004)$; These differences are statistically significant, especially in the cases of GBP4, EGFR and TTF1 proteins (see Figs. 3 and 5).

It is known that IE protein is expressed within a few minutes after HCMV infection [author: please include a reference here]. Regardless of whether it is expressed in brain metastases or the primary lung tumor, or whether it is caused by HCMV infection, it is important to note that most of the cells are not just infected, and that only replicating cells that have just been infected by the new generation of HCMV may be positive. The results we obtained in this study are consistent with these observations. The immune complexes present in cells recently infected by HCMV are of granular nature and are located in the cytoplasm of the cell, suggesting that the virus broke the membrane and entered the cell, as shown in Case No. 2 and 3 in Fig. 6. As for the EGFR protein, Song [7] described its characteristics in R language in the immune microenvironment of the brain metastases of lung cancer. We found that mutant EGFR protein tends to cluster together in the brain, in a pattern that is different from the one resulting from the up regulation of various immune-related pathways of brain metastases. No up-regulation was seen in the primary tumors. These results are consistent with the ones reported in previous studies [7] (see Fig. 7).

\section{Discussion}

Based on our literature search performed in PubMed, the first reports on brain metastases of lung cancer date back to 1981 . Since 2014, 20-30 studies have been published every year. It is important to conduct in-depth research in this largely unexplored field. Yousefi et al. [9] claimed that targeted therapy was most necessary, unfortunately, however, the lack of understanding of the molecular mechanisms underlying brain metastases has hampered the development of this area of research. It is well recognized that lung cancer preferentially metastasizes to the brain, where it interacts with other molecules present within the tissue microenvironment to form a complex biological network that supports the survival and proliferation of cancer cells [10]. CXCL12 and its receptor CXCR4 have long been the target of research of brain metastases of lung cancer. Paratore et al. [11] reported that immune response of cavallo, CXCR4 and CXCL12 can help distinguish primary non-small cell lung cancer with or without brain metastases. Along the same lines, Chen et al.[12] suggested that the high level of CXCR4 expression was related to brain metastases of non-small cell lung cancer. However, the role of TTF1, which was previously considered to be a specific marker of brain metastases of lung cancer, remains controversial. Unal et al. [13] claimed that although TTF1 was highly expressed in of brain metastases of lung cancer, it is also expressed in primary gliomas. Therefore, its specificity brain metastases of lung cancer are questionable. In our study, except for Case No. 5, TTF1 was highly expressed in both the primary tumor and metastases. 
So far, EGFR-related research [7] has mainly discussed the issue of tumor immunity, and has not yet explored the relationship between cancer and HCMV infection. In this study, we aimed to further our understanding of the links between brain metastases and HCMV infection. Although preliminary, our results suggest a potentially important effect resulting from the combination of IE and GBP4 protein expressions.

To the best of our knowledge, no relevant reports on the role of GBP4 in brain metastases of lung cancer have been reported. However, it is known that GBP4 is a GTPase protein induced by IFN-g and is essential for the activation of inflammasomes. It is also known that CXCL8 and LTB4 of the inflammasome play a role in the recruitment of neutrophils to the site of infection, where pathogens are then eliminated by prostaglandin D2 [14]. The expression of tumor suppressing genes is affected in brain metastases, nevertheless, in seven samples of the five cases of brain metastases of lung cancer reported in this study we found high expression of the GBP4 protein. How to explain these findings is an important endeavor. $\mathrm{Xu}$ et al. [15] reported that the high expression of GBP3 protein observed in glioma is involved in the regulation of the malignant progression of glioma cells by activating p62. For this reason, it is worth speculating whether, in the cases we have analyzed, brain metastases were facilitated by the action of GBP4 through CXCR4.

With regards to the IE protein, it expression is commonly found in cancer patients infected with HCMV. Halwachs et al. [16] reported that gene expression [author: gene expression of what? Please clarify which genes/pathways] after HCMV infection occurs in a transient cascade, and includes three phases: major immediate-early (MIE), early ( $E)$ and late $(L)$ phases. Briefly, after the virus envelope fuses with the plasma membrane of the host cell, the enveloped virus particles are released into the cytoplasm, enter the nucleus through the nuclear pore within a few minutes and release viral DNA triggering the different phases of gene expression. Dooley et al. [17] reported that after HCMV infection, through the combined action of chromatin remodeling and transcription factors, the genes expressed in the MIE phase play an important regulatory role in the balance between latent infection and lytic infection. This molecular switch involves the action of a large number of host and viral proteins. In addition to the host-encoded MIE site regulatory factors, HCMV also encodes proteins such as UL138 [18] and US28 [19] during the incubation period, in order to inhibit immune responses that results in MIE transcription. Currently, only a number of the molecules involved in these processes are known, and our knowledge of this topic is still very limited. The results of this study show that, overall, the levels of IE expression are low in all tested samples, although there were also a few cells where the immune complexes were dark brown and granular, which is in line with the high expression of viral DNA characteristics, as shown in Fig. 6. Hence, IE positive expression in these few cells may be caused by HCMV infection in the MIE stage. The fact that the cases we analyzed are in the late stage of brain metastases of lung cancer, and therefore most of the cells infected by HCMV should be in the late stage of $E$ and L, may be the explanation why only a few positive IE cells were observed. As mentioned above, GBP4 protein may be a member of a large number of host and viral proteins involved in these mechanisms. In the E and L phases after HCMV infection, a large amount of HCMV is required to participate in DNA replication. Therefore, among the five proteins, the expression of GBP4 is the strongest and the most extensive. 
Targeted therapy of brain cancer of lung metastases, including targeted therapy that inhibits EGFR, can increase the median survival time to 12 months [20]. Using TKIs (EGFR-specific tyrosine kinase inhibitors) to treat brain cancer of lung metastases with EGFR mutations can prolong the median survival time to 15-20 months, with a remission rate of 60\%-100\% [21]. Inhibitors include Erlotinib[22], Erbitux [23], Gefitinib [24] and mouse monoclonal (mab) 225 and 528 [25]. Unfortunately, drug resistance cases following the above-mentioned target molecule treatment is a common occurrence. As suggested in Table 1, Fig. 4 and Fig. 5, if patients with brain cancer of lung metastases are infected by HCMV, GBP4 inhibitors may be more effective given that GBP4 protein expression appear broader than EGFR expression.

Research of the mechanisms underlying brain cancer of lung metastases is a hot topic of research that remains largely unexplored. This study contributes to the existence knowledge in this area. The limitation of this study is that the number of cases is too small and the detection indicators are single. Our findings should be confirmed in larger studies.

\section{Abbreviations}

HCMV

human cytomegalovirus; IE:immediate early gene; GBP4:guanine nucleotide-binding protein 4; CXCR4:CXC chemokine receptor 4; TTF1:thyroid transcription factor 1; EGFR:epidermal growth factor receptor; AOD:average optical density;PBS:phosphate buffered saline; H\&E:Hematoxylin and eosin.

\section{Declarations}

\section{Acknowledgements}

Not applicable.

\section{Authors' contributions}

JW, and HFZ performed the experiments, analyzed the data and drafted the manuscript. WYZ and ZMW designed the study and revised the manuscript. QH revised the manuscript. KY, XTX, DHG, WHW and XFF performed the experiments.

\section{Funding}

This work was supported by the Medical-Health Science- Technology Plan of Suzhou New District (No. 2018Q010) and the People's Livelihood Science and Technology Project of Suzhou Science and Technology Administration (No. SYS2020078).

\section{Availability of data and materials}


The datasets used and/or analyzed during the current study are available from the corresponding author on reasonable request.

\section{Declarations}

\section{Ethics approval and consent to participate}

This study was approved by the Ethics Committee of the Affiliated Suzhou Science \& Technology Town Hospital of Nanjing Medical University (Suzhou, China). The processing of clinical tissue samples is in strict compliance with the ethical standards of the Declaration of Helsinki. All patients signed written informed consent.

\section{Consent for publication}

Consent for publication was obtained from the participants.

\section{Competing interests}

The authors declare that they have no competing interests.

\section{References}

1. Bowman KM, Kumthekar P. Medical management of brain metastases and leptomeningeal disease in patients with breast carcinoma. Future Oncol. 2018;14:391-407.

2. Rusu-Cordunean F, Berlea ML, Cernomaz AT, Marinca MV, Peter S, Pavel I, Grigoriu BD. Egfr Mutations in Non-Small Cell Lung Cancer: Local Epidemiology and Clinical Importance. Rev Med Chir Soc Med Nat lasi. 2015;119:1031-6.

3. Taher C, Frisk G, Fuentes S, Religa P, Costa H, Assinger A, Vetvik KK, Bukholm IR, Yaiw KC, Smedby $\mathrm{KE}$, et al. High prevalence of human cytomegalovirus in brain metastases of patients with primary breast and colorectal cancers. Transl Oncol. 2014;7:732-40.

4. Farias K, Moreli ML, Floriano VG, da Costa VG. Evidence based on a meta-analysis of human cytomegalovirus infection in glioma. Arch Virol. 2019;164:1249-57.

5. Groblewska M, Litman-Zawadzka A, Mroczko B. The Role of Selected Chemokines and Their Receptors in the Development of Gliomas. Int J Mol Sci 2020, 21.

6. Haque M, Siegel RJ, Fox DA, Ahmed S. Interferon-stimulated GTPases in autoimmune and inflammatory diseases: promising role for the guanylate-binding protein (GBP) family. Rheumatology. 2021;60:494-506.

7. Song SG, Kim S, Koh J, Yim J, Han B, Kim YA, Jeon YK, Chung DH. Comparative analysis of the tumor immune-microenvironment of primary and brain metastases of non-small-cell lung cancer reveals organ-specific and EGFR mutation-dependent unique immune landscape. Cancer Immunol Immunother 2021. 
8. Jerome Marson V, Mazieres J, Groussard O, Garcia O, Berjaud J, Dahan M, Carles P, Daste G.

Expression of TTF-1 and cytokeratins in primary and secondary epithelial lung tumours: correlation with histological type and grade. Histopathology. 2004;45:125-34.

9. Yousefi M, Bahrami T, Salmaninejad A, Nosrati R, Ghaffari P, Ghaffari SH. Lung cancer-associated brain metastasis: Molecular mechanisms and therapeutic options. Cell Oncol (Dordr). 2017;40:41941.

10. Spano D, Zollo M. Tumor microenvironment: a main actor in the metastasis process. Clin Exp Metastasis. 2012;29:381-95.

11. Paratore S, Banna GL, D'Arrigo M, Saita S, lemmolo R, Lucenti L, Bellia D, Lipari H, Buscarino C, Cunsolo R, Cavallaro S. CXCR4 and CXCL12 immunoreactivities differentiate primary non-small-cell lung cancer with or without brain metastases. Cancer Biomark. 2011;10:79-89.

12. Chen G, Wang Z, Liu XY, Liu FY. High-level CXCR4 expression correlates with brain-specific metastasis of non-small cell lung cancer. World J Surg. 2011;35:56-61.

13. Unal B, Yildirim S, Sezer C, Suren D. TTF-1 may not be a reliable marker for differentiating metastasis from brain tumors. Turk Patoloji Derg. 2014;30:201-5.

14. Tyrkalska SD, Candel S, Angosto D, Gomez-Abellan V, Martin-Sanchez F, Garcia-Moreno D, ZapataPerez R, Sanchez-Ferrer A, Sepulcre MP, Pelegrin P, Mulero V. Neutrophils mediate Salmonella Typhimurium clearance through the GBP4 inflammasome-dependent production of prostaglandins. Nat Commun. 2016;7:12077.

15. Xu H, Sun L, Zheng Y, Yu S, Ou-Yang J, Han H, Dai X, Yu X, Li M, Lan Q. GBP3 promotes glioma cell proliferation via SQSTM1/p62-ERK1/2 axis. Biochem Biophys Res Commun. 2018;495:446-53.

16. Halwachs-Baumann G. Recent developments in human cytomegalovirus diagnosis. Expert Rev Anti Infect Ther. 2007;5:427-39.

17. Dooley AL, O'Connor CM. Regulation of the MIE Locus During HCMV Latency and Reactivation. Pathogens 2020, 9.

18. Montag C, Wagner JA, Gruska I, Vetter B, Wiebusch L, Hagemeier C. The latency-associated UL138 gene product of human cytomegalovirus sensitizes cells to tumor necrosis factor alpha (TNF-alpha) signaling by upregulating TNF-alpha receptor 1 cell surface expression. J Virol. 2011;85:11409-21.

19. Zhu D, Pan C, Sheng J, Liang H, Bian Z, Liu Y, Trang P, Wu J, Liu F, Zhang CY, Zen K. Human cytomegalovirus reprogrammes haematopoietic progenitor cells into immunosuppressive monocytes to achieve latency. Nat Microbiol. 2018;3:503-13.

20. Shepherd FA, Rodrigues Pereira J, Ciuleanu T, Tan EH, Hirsh V, Thongprasert S, Campos D, Maoleekoonpiroj S, Smylie M, Martins R, et al. Erlotinib in previously treated non-small-cell lung cancer. N Engl J Med. 2005;353:123-32.

21. Baselga J. The EGFR as a target for anticancer therapy-focus on cetuximab. Eur J Cancer. 2001;37(Suppl 4):16-22.

22. Fukuoka M, Yano S, Giaccone G, Tamura T, Nakagawa K, Douillard JY, Nishiwaki Y, Vansteenkiste J, Kudoh S, Rischin D, et al. Multi-institutional randomized phase II trial of gefitinib for previously 
treated patients with advanced non-small-cell lung cancer (The IDEAL 1 Trial) [corrected]. J Clin Oncol. 2003;21:2237-46.

23. Sato JD, Kawamoto T, Le AD, Mendelsohn J, Polikoff J, Sato GH. Biological effects in vitro of monoclonal antibodies to human epidermal growth factor receptors. Mol Biol Med. 1983;1:511-29.

24. Wu YL, Zhou C, Cheng Y, Lu S, Chen GY, Huang C, Huang YS, Yan HH, Ren S, Liu Y, Yang JJ. Erlotinib as second-line treatment in patients with advanced non-small-cell lung cancer and asymptomatic brain metastases: a phase II study (CTONG-0803). Ann Oncol. 2013;24:993-9.

25. Costa DB, Shaw AT, Ou SH, Solomon BJ, Riely GJ, Ahn MJ, Zhou C, Shreeve SM, Selaru P, Polli A, et al. Clinical Experience With Crizotinib in Patients With Advanced ALK-Rearranged Non-Small-Cell Lung Cancer and Brain Metastases. J Clin Oncol. 2015;33:1881-8.

\section{Tables}

Table 1. The proteins involved in the routine pathology and the five proteins we studied

\begin{tabular}{|c|c|c|c|c|c|c|c|}
\hline & Case 1 & Case 1 & Case 2 & Case 2 & Case 3 & Case 4 & Case 5 \\
\hline & Brain & Lung & Brain & Lung & Brain & Brain & Brain \\
\hline 1.IE & + & $H$ & + & \pm & + & + & + \\
\hline 2.GBP4 & + & $+1+$ & H+ & $H$ & H+ & $+1+$ & $H+$ \\
\hline 3.CXCR4 & \pm & \pm & H & \pm & H+ & + & H \\
\hline 4.CD68 & 0 & 0 & 0 & 0 & 0 & 0 & + \\
\hline 5.CD56 & - & 0 & + & + & 0 & 0 & - \\
\hline 6.CD99 & 0 & 0 & part + & 0 & - & - & 0 \\
\hline 7.NSE & 0 & 0 & + & 0 & part + & part + & 0 \\
\hline 8.Syn & 0 & - & 0 & + & 0 & - & - \\
\hline 9.GFAP & - & 0 & - & 0 & - & - & part + \\
\hline $10 . \mathrm{CK}$ & + & & + & + & part + & part + & + \\
\hline $11 . \mathrm{CK} 7$ & + & + & a few + & + & part + & part + & scattered + \\
\hline 12.CEA & 0 & 0 & 0 & 0 & 0 & \pm & \\
\hline 13.P53 & 0 & 0 & + & 0 & 0 & 0 & part + \\
\hline 14.TTF1 & ++ & +++ & ++ & +++ & +++ & +++ & - \\
\hline 15.EGFR & ++ & + & ++ & - & - & + & +++ \\
\hline 16.EMA & + & 0 & 0 & 0 & part + & part + & a few + \\
\hline 17.HER2 & 0 & 0 & 0 & 0 & 0 & +++ & +++ \\
\hline 18. NapsinA & + & + & $=$ & 0 & $=$ & + & - \\
\hline $19 . \mathrm{Ki}-67$ & $60 \%+$ & $60 \%+$ & $70 \%+$ & $\begin{array}{c}* 95 \%+ \\
15 \%+\end{array}$ & $60 \%+$ & $30 \%+$ & $30 \%+$ \\
\hline
\end{tabular}

"small cell carcinoma $95 \%+$, adenocarcinoma $15 \%+; 0$ not tested , - negative , \pm weakly positive , + positive , ++ medium positive ,

\section{Figures}



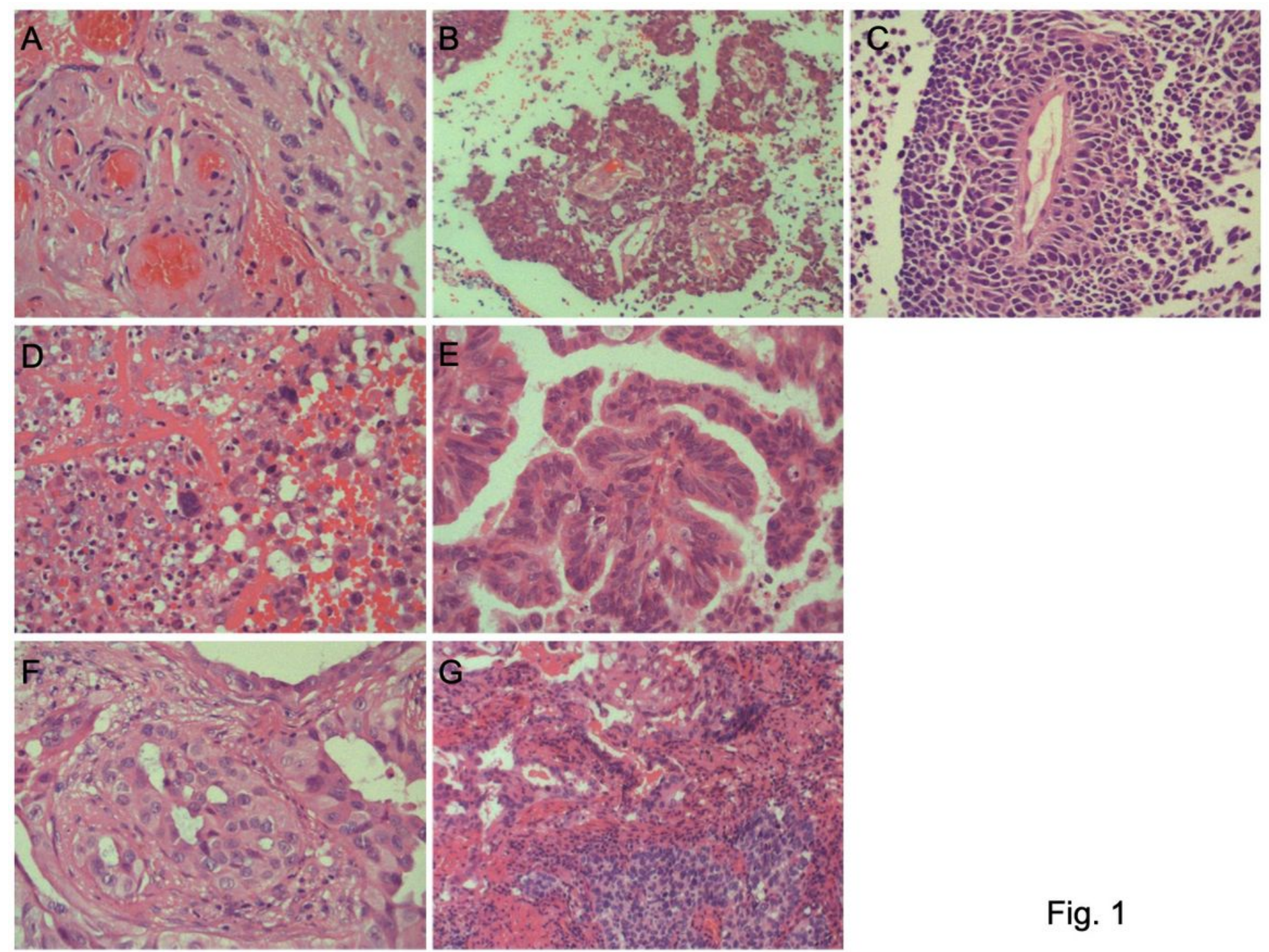

Fig. 1

\section{Figure 1}

H \& E staining light microscopic (X400): A - E were brain metastases in cases 1 - 5, F and $G$ were primary lung lesions in cases 1 and 2, respectively, A (case 1) was adenocarcinoma; B (case 2) was small cell carcinoma; C (case 3 ) was poorly differentiated adenocarcinoma; D (case 4) was papillary invasive carcinoma; $E$ (case 5) was adenocarcinoma; $F$ and $G$ were primary lung lesions, which were adenocarcinoma and mixed carcinoma respectively $(G$, adenocarcinoma on the white line, while small cell carcinoma below it which is labeled artificially) 


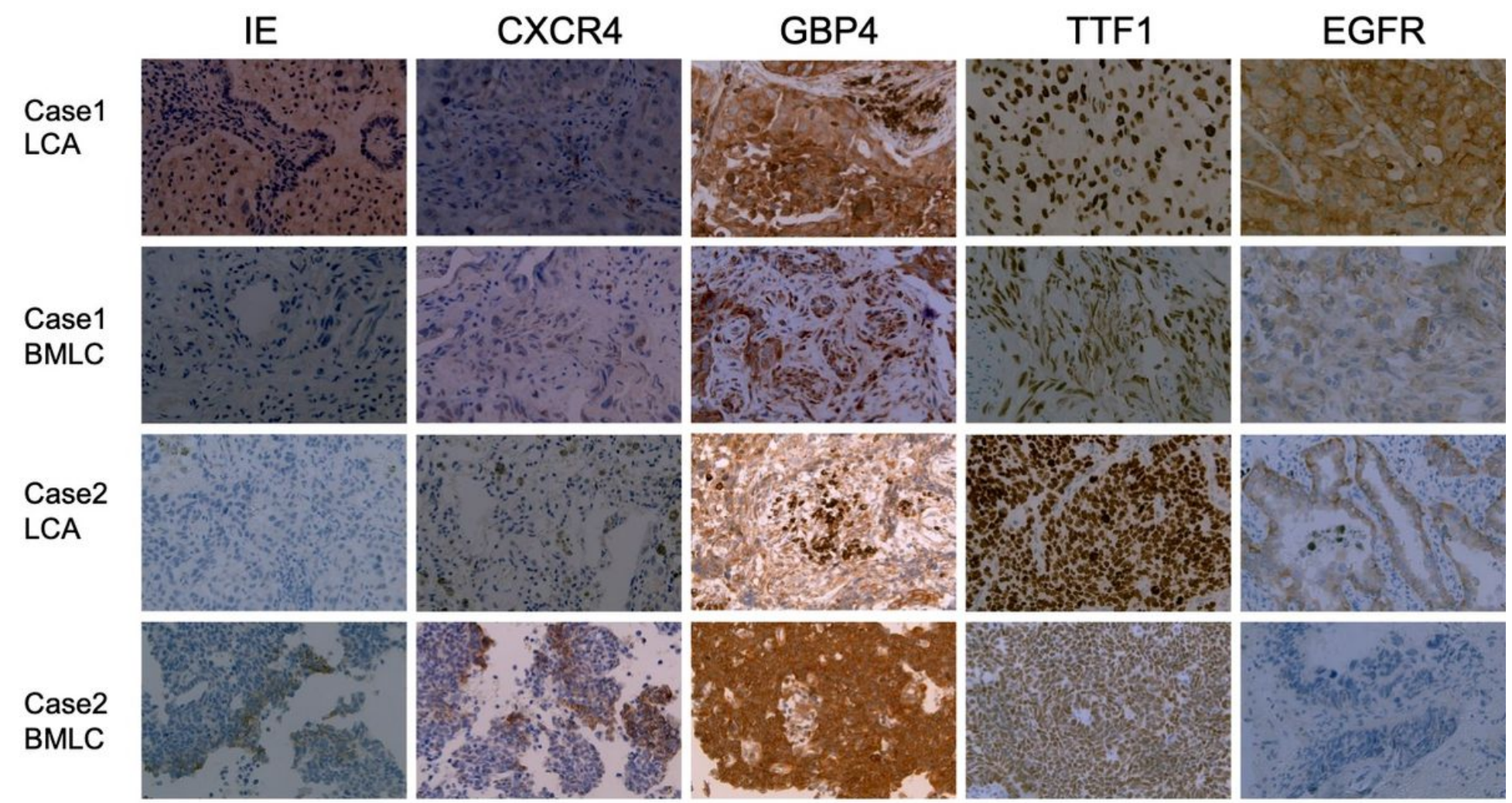

Fig. 2

Figure 2

The immunohistochemical light microscopic of five target proteins $(\times 400)$ from primary foci (LCA) and metastatic foci (BMLC) of case 1 and case 2, respectively. Primary foci of case 1 in the first line (lung), and metastatic foci of case 1 in the second line (brain); primary foci of case 2 in the third line (lung), metastatic foci of case 2 in the fourth line (brain). According to the depth of immune complex staining in different parts of the same case, the difference between primary lesions and metastatic lesions was evaluated by the conversion of mean optical density $(A O D)(P<0.05)$. The statistical results of $A O D$ are shown in Figure 3. 

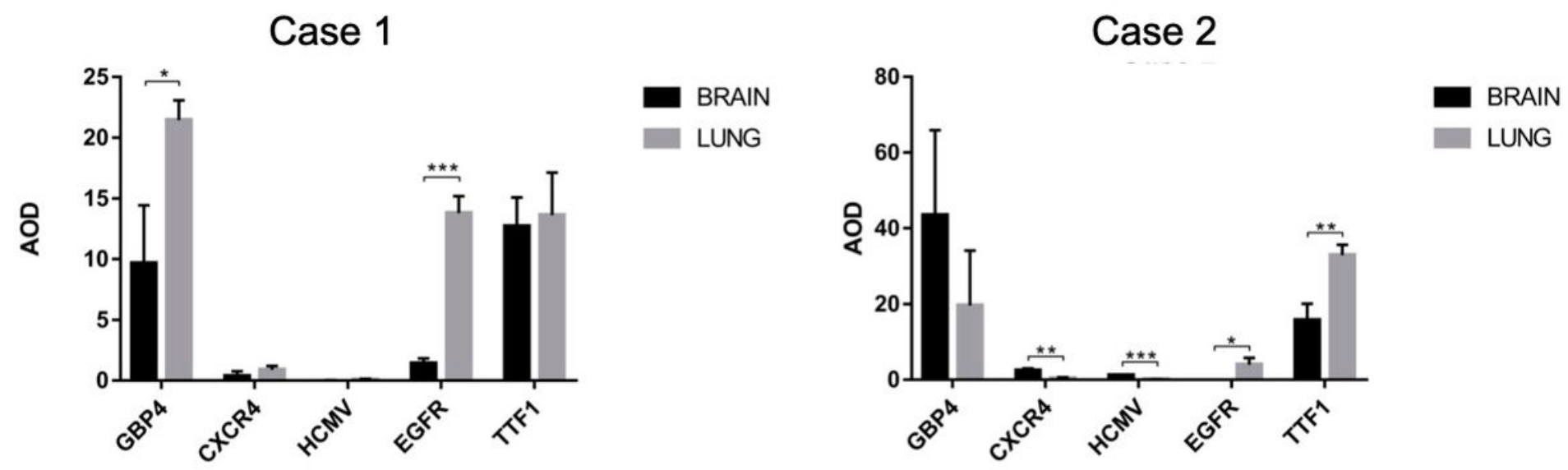

Fig. 3

\section{Figure 3}

Comparison of VOD values of five target proteins in case 1 and case2 about primary and metastatic lesions: in case1, the differences of GBP4 and EGFR was significant $(P<0.05)$; in case 2, the differences of CXCR4, HCMV, EGFR and TTF1 were also significant $(P<0.05)$. These differences are statistically significant, especially in the cases of GBP4, EGFR and TTF1 proteins. ${ }^{*} p<0.05,{ }^{\star \star} p<0.01$, ${ }^{\star \star \star} p<0.001$. 


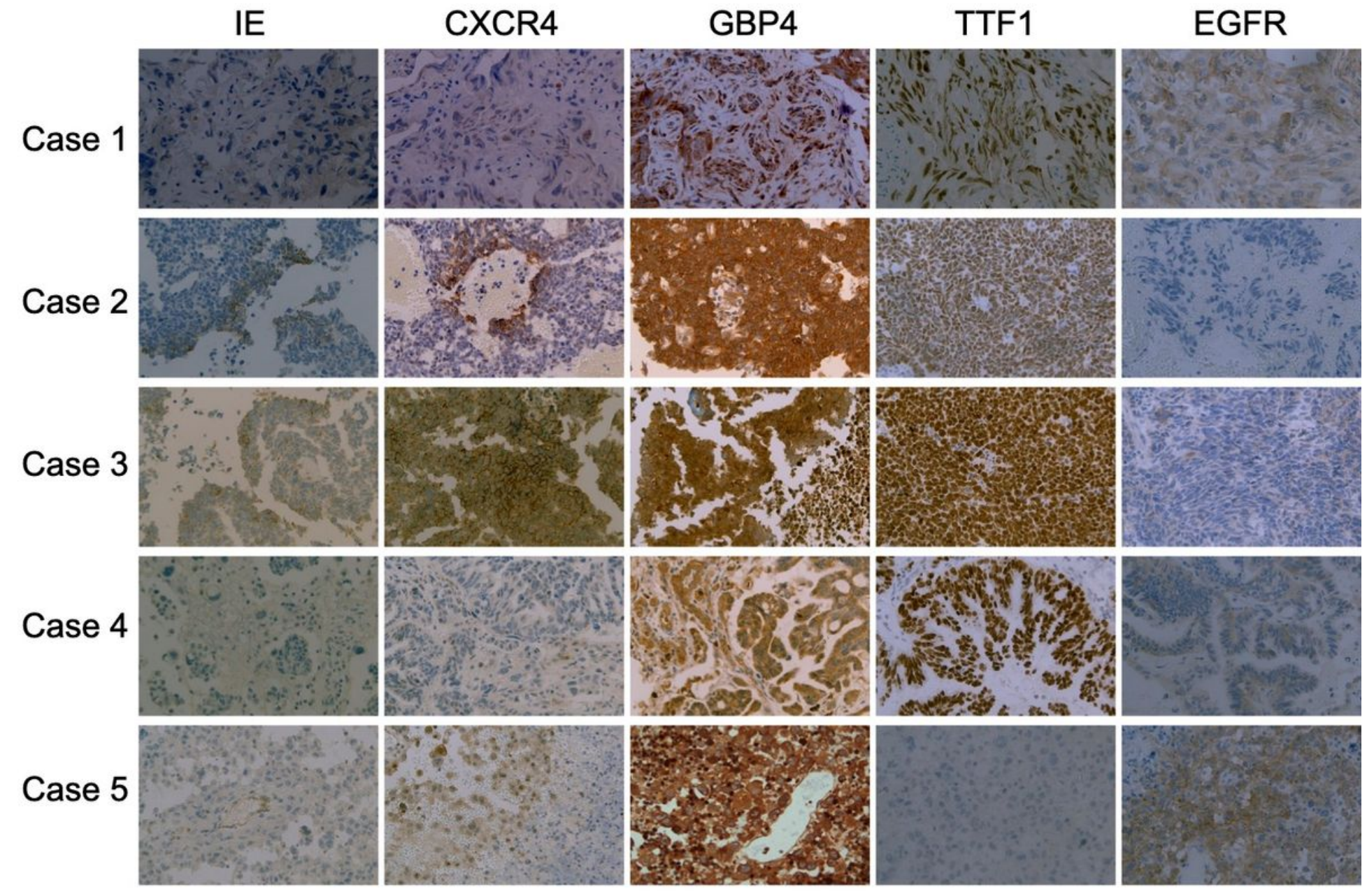

Fig. 4

\section{Figure 4}

The immunohistochemical light microscopic of five target genes expression in brain metastases $(\times 400)$ : the expression intensity was evaluated by the depth of immune complex staining. The statistics of average optical density (AOD) is shown in Fig. 5. 

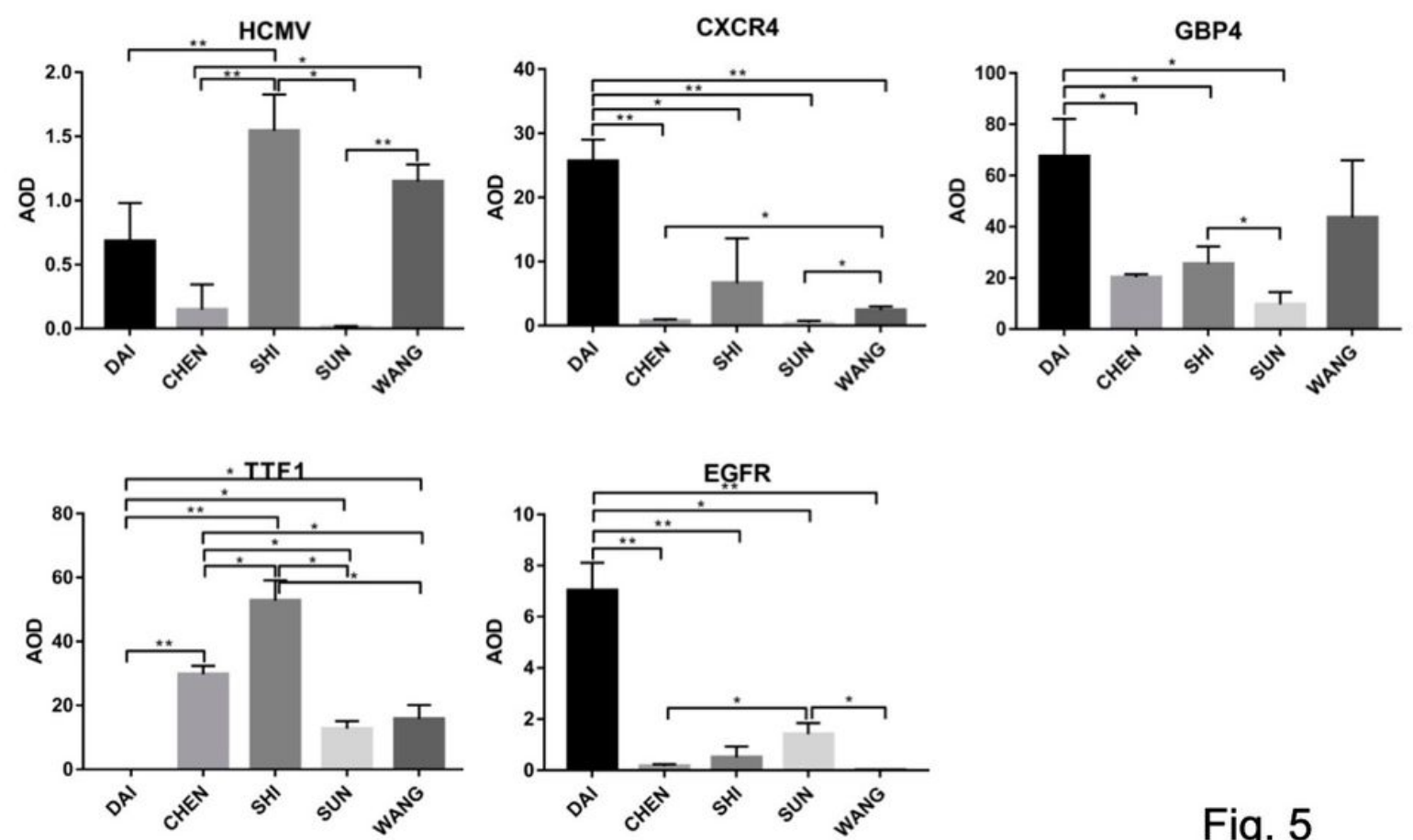

Fig. 5

\section{Figure 5}

The average optical density (AOD) of HCMV, CXCR4, GBP4, TTF1 and EGFR in brain metastases. The expressions of HCMV, CXCR4, TTF1 and EGFR were significantly different in different metastatic foci, while the expression of gbp4 was higher in all tissues, and the difference was smaller than the other four proteins. ${ }^{\star} p<0.05,{ }^{*} \mathrm{p}<0.01,{ }^{*} * \mathrm{p}<0.001$. 

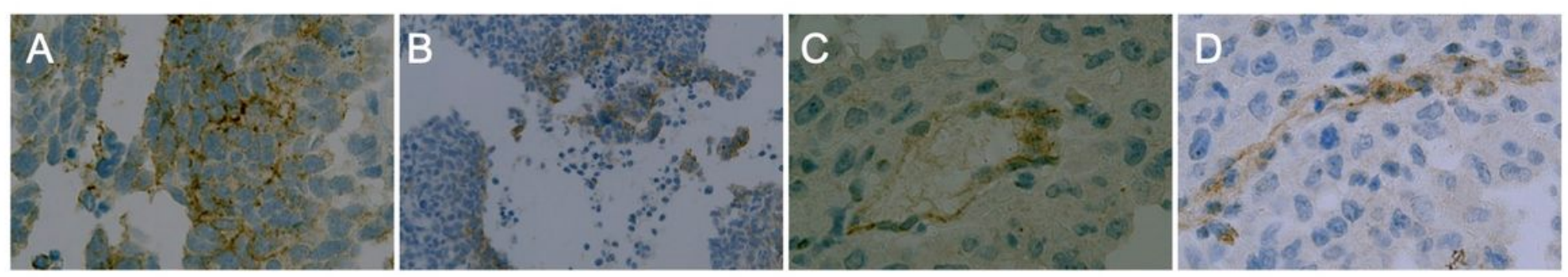

Fig. 6

\section{Figure 6}

Light microscopic observation of IE in brain metastases ( $\times 1000)$ : most of the dark brown immune complexes with viral characteristic DNA particles were located in the cell membrane (A and B), a few in the cytoplasm or along the blood vessels (C and D). 

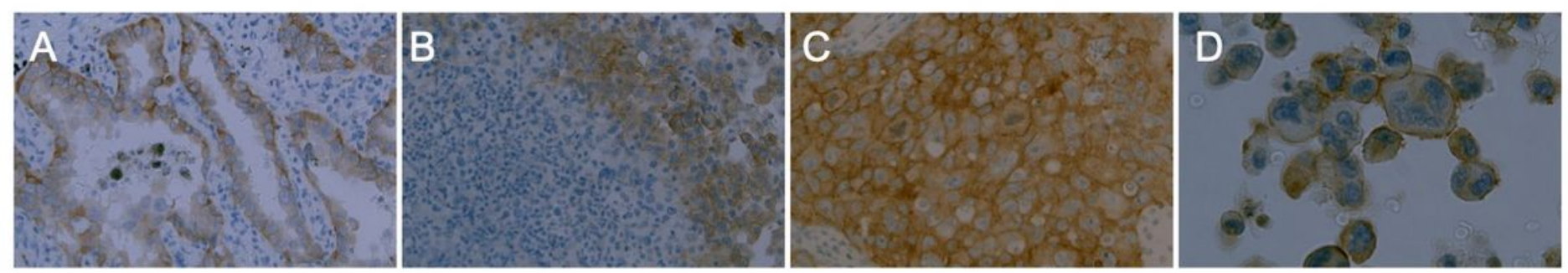

Fig. 7

\section{Figure 7}

Expression of EGFR in primary and metastatic lesions. A. Low expression in primary lung lesions $(\times 400)$; $B-D$. Highly expression in metastatic lesions: positive cells gathered together $(B(\times 400))$, immunocomplex localization of cell membrane and intercellular matrix $(C(\times 400))$, The stained cells vary in size and shape, and some of them have multiple nuclei (D. $(\times 1000)$ ). 